\section{Original sirticles.}

\section{SOME CASES ILLUSTRATING CYSTOSCOPIC DIAGNOSIS.}

By ARTHUR L. CHUTE, M.D., BOstoN.

To many, cystoscopy seems a needless refinement of diagnosis, giving discomfort to the patient and incapable of accurate results. The wider my experience becomes, the more I am impressed with the accuracy of cystoscopy as a diagnostic measure as well as its painlessness and lack of serious after effects. From among the cases seen in the last few months the following have been selected as good illustrations of the help cystoscopy may render in the diagnosis of urinary cases. In none of these cases has a general anesthetic been used. When the patients have complained it has been of the irksomeness of the position rather than of the pain caused by the examination.

CASE I is that of a young man, twenty-one, who presented a congenital diverticulum of the bladder. He had never had any venereal disease. Three weeks before he presented himself at the dispensary clinic he began, without cause, to have frequent micturition. His urine became turbid; the frequency increased and urination became painful. At one time his urine was moderately bloody. The ordinary treatment for cystitis had given no relief. Examination, May 5, 1905 , showed a very turbid urine containing a slight trace of albumin. There was a considerable sediment made up of a little normal blood, a great deal of pus, much bladder epithelium and many bacteria. No renal elements were seen. His kidneys were not palpable, nor were they tender. His prostate as well as his testes and epididymes were normal. Considerable difficulty was found in washing his bladder clean; it was, however, moderately tolerant to distention. The cystoscope showed a normal bladder base. On the posterior bladder wall in a line with the center of the interureteric ligament and above it, there was an elliptical opening about three fourths of an inch in its greatest diameter. Fluid containing particles of pus and mucus could be seen washing in and out with each deep inspiration. Abdominal pressure would cause fluid to gush out in large amounts. The upper part of the posterior wall of the diverticulum could be seen as it was pushed toward the opening during inspiration; it had a roughened surface covered with shreds of muco-pus. The light, shining from behind, through the septum between the diverticulum and the bladder, gave a very beautiful picture of the engorged vessels in this partition.

The patient refused operative treatment and gradually quieted down under palliative measures.

As there was no history of trauma or of urethral obstruction in this patient, it is probable that we had to do with a congenital defect in the muscular layer of the bladder wall, through which a gradual protrusion of mucous membrane had taken place. As this protrusion of mucous membrane increased it had sagged down until a small pouch had formed that could not be emptied during micturition. The patient had a residual urine in this pouch. Organisms entered the cavity either by way of the kidneys or the lymphatics and set up an acute inflammatory process.

It is to be expected that this patient will suffer from repetitions of this, and that each inflammatory attack will, by making the adhesions between this sac and the surrounding tissues firmer, render the radical cure of the diverticulum more difficult.

CASE II presents a diverticulum of different origin. The patient, age seventy-nine, was operated upon for prostatic obstruction five years ago. The operation by the suprapubic route was evidently only a partial enucleation. He had a perineal section, presumably for stricture some years before. Since his suprapubic operation his urine had always been turbid, and he had been subject to causeless exacerbations, during which his urine became ammoniacal and he had suprapubic tenderness, pain and marked frequency. Usually he had no residual; occasionally as much as an ounce.

Cystoscopic examination showed jets of fairly clear urine from both ureters, but with a little puffiness about the right orifice. The bladder showed some trabeculation, with a dull, white, thickened mucous membrane. There was a small bas fond. The prostate showed a slight median enlargement with a moderate bulging into the bladder of both lateral lobes, more particularly the right. On the anterior surface of the bladder, just to the right of the median line, and in a position that corresponded with his suprapubic scar, there was an oblong opening one-half inch long. Turbid urine containing flakes of pus could be seen going in and out of this opening during respiration. Pressure on the abdominal wall caused the diverticulum to gape open.

This diverticulum was undoubtedly due to a weak spot in the muscular coat of the bladder that followed the suprapubic incision. It had allowed a pocket-like protrusion of the mucous membrane to take place. As this pocket was not properly drained it had acted as a storehouse for organisms that had periodically reinfected his bladder. Because of his age and the fact that with care he could be kept fairly comfortable no operation was advised. The cystoscopic examination, however, afforded a satisfactory explanation for the continuance of his bladder infection in the absence of a residual urine, and for his recurring attacks of cystitis.

It is not evident just why there should have been a protrusion of the scar in the bladder wall of a patient who emptied his bladder so completely as this man did, and in whom there had been so little back pressure.

CASE III is that of a woman of thirty-eight with a prolapsed right kidney. She presented herself at St. Elizabeth's Hospital in November, 1905. Her chief complaint was of pain in her right loin when she had to stand any length of time. She had, beside this pain, backache, due apparently to a slight grade of atrophic arthritis of the spine, also vague neurasthenic symptoms, so that I was inclined at first to put but little importance on a pretty freely movable right kidney that was only very slightly tender. As treatment of the arthritic trouble and improved general health did not give relief to her right lumbar pain, I was led to investigate her right kidney as carefully as possible to determine what part it played in her pain. Examination of a catheter specimen of her bladder urine showed that it was not quite transparent and that it contained a very slight trace of albumin; there was a little sediment made up of a lew leucocytes and a considerable number of granular epithelial cells. The urine showed no organisms in cover slip preparations. Cystoscopic examination of the bladder itself showed no abnormality. A catheter introduced a short dis- 
tance into the left ureter showed a transparent, nonalbuminous urine without sediment, but a specimen from the right ureter contained albumin in very slight amount and granular cells and leucocytes such as were found in the bladder urine; the catheter passed to the right kidney pelvis showed a slight amount of aseptic renal retention. Filling the right kidney pelvis with sterile boric solution by means of the ureter catheter exaggerated the pain she complained of in her right loin. A second examination after the patient had been in bed instead of on her feet showed no retention in the kidney pelvis.

The epithelium coming wholly from one kidney, the aseptic renal retention when the patient had been on her feet and its absence when she had been in bed, as well as the pain on distending the renal pelvis, all seemed to point pretty definitely to the movable kidney as the cause of her right lumbar pain. The right kidney was cut down upon and fixed in place. The patient was seen a month later, at which time there had been no return of her pain, but too short a time had elapsed to tell whether or not this relief would be permanent.

Beside the pain in this prolapsed kidney, which was undoubtedly due to dragging on the pedicle, it is interesting to note that we had an aseptic renal retention that had begun to cause changes in the renal pelvis. These changes were apparently a maceration and desquamation of the pelvic epithelium, - changes that would probably have made the organ particularly susceptible to infection. This aseptic renal retention does not exist in all prolapsed kidneys, but when it is present it should be considered as an extra-indication for fixing the kidney in a position that will facilitate its drainage.

CASE IV. A man, forty-three, with a septic retention of his left kidney pelvis. This man came to the Boston Dispensary in the fall of 1905 in the service of Dr. C. M. Whitney, through whose courtesy I saw him. Though he denied venereal infection, it seems possible that he may have been mistaken as to this. His trouble began eleven years ago with a slight urethral discharge, which was followed by a subacute epididymitis, according to his story. Shortly after this time he began to have backache, suprapubic pain and pain in his groins. Since about 1900 he had had a turbid urine with nocturnal frequency (four or five times). He had little pain on micturition. For two years he had had bladder irrigations without effect. When he first appeared at the clinic he showed a chronic prostatitis and vesiculitis. His urine, which was very turbid, did not, however, clear with the treatment of his prostate. Neither kidney was tender nor palpable. Cystoscopic examination showed a tolerant bladder with a slight cystitis of the base. There was a curious hood-like puckering high up on the posterior wall which may have been congenital in origin or due to some former ulceration. The right ureter was on a considerable papilla; it was normal in appearance and emitted unusually strong and frequent spurts of clear urine. The left ureter was also on a papilla; it was "slipper shaped" and a bit of muco-pus could be seen protruding from the ureteric orifice. Pushing against the posterior bladder wall with the beak of the cystoscope made the ureter gape open and assume the appearance of the " golf hole " ureter of Fenwick. A catheter passed into the left ureter very easily and, left in place for twenty minutes, gave but a few drops of turbid fluid; when passed to the kidney pelvis, the catheter excavated 2 oz. of turbid fluid in a short time. This fluid contained a trace of albumin, $.50 \%$ of urea, a little blood, some epithelium, a large amount of pus and a great number of bacteria. No tubercle bacilli could be found in cover slip preparations. A catheter specimen from the right ureter showed a transparent urine of normal color containing a slight trace of albumin, urea $2.27 \%$. The sediment, which was very slight, showed a single cast, a little normal blood and a few epithelial cells, the blood and epithelial cells probably due to a slight injury of the ureteral membrane by the catheter. The ureteric jets on the right were at times as frequent as every five seconds and occasionally there would be twenty to thirty drops in a jet, showing a very actively secreting organ.

This man was under observation for a considerable time and repeated examinations gave practically the same results. Washing out his left kidney pelvis made his urine considerably clearer temporarily and lessened his nocturnal frequency. He should submit to kidney drainage or nephrectomy, which was advised him.

This patient, who had been supposed to be suffering from a cystitis for which he had received bladder irrigations for two years, illustrates in a striking way the extent to which a renal suppuration may occasionally go without giving symptoms that call particular attention to the kidney; also the fallacy of expecting to find a palpable or tender kidney in all renal suppurations. At no time was I able to detect any mass or tenderness in his left loin.

CASE V. A woman of thirty-nine, referred for examination in December, 1905, by Dr. H. Lincoln Chase. She had been delivered of her eighth child seven weeks before. Immediately after delivery Dr. Chase had noticed a considerable mass in her right lumbar region, which was elastic and the shape of an enlarged kidney. It was movable and could be separated from the liver above. This mass gave considerable pain for the first three weeks following labor; since then it had been painless, though it had gradually increased in size. A week before she was seen she suddenly became blind in her left eye, due, it is said, to a retinal hemorrhage: The blindness persisted at the time of examination, though it was clearing up some when this woman was last seen.

Her urine, which was very slightly turbid, contained leucocytes in small numbers and epithelial cells. Tubercle bacilli were found in it. The patient occupied the same room with her husband, who had advanced phthisis, and the presumption was that she had a tuberculosis of her right kidney. The bladder, which was perfectly tolerant, showed a little redness about the left ureter. The right ureteric orifice was small and normal looking. A No. 6 catheter could be introduced into the right ureter about one and one half inches, but could not be made to go any further. No urine could be gotten. A catheter passed into the left ureter perfectly easily, and a half ounce of urine, of about the same turbidity as the bladder specimen, was obtained, in a short time. In this urine tubercle bacilli were found. At a subsequent examination, three weeks after the first, the findings were the same.

The knowledge gained in this case was of considerable importance and raised several interesting points. On the one hand we found that the supposedly well kidney was not an entirely sound one, and while it was at that time perfectly competent it could not be depended upon 
to continue so for any length of time. On the other hand the finding of tubercle bacilli in the left kidney, together with the obstruction low down in the right ureter, raised the hope that the condition in the right kidney might be purely an hydronephrosis due to an impacted calculus, and that if relieved before too long a time elapsed, the kidney might be capable of a certain amount of work and of helping out to some extent the kidney known to be tuberculous. The patient has not as yet been willing to submit to operation.

CAse VI. An Italian woman of about thirty, who entered St. Elizabeth's Hospital in November, 1905, in the service of Dr. Kingman, to whom I am indebted for the privilege of reporting the case. She spoke but little English, and her complaint, so far as could be learned, was of backache and dysuria. She had a heavy uterus in the third degree of retroversion, with nothing else that could be made out on pelvic examination. Her urine showed the characteristics of a chronic cystitis. It was supposed that her pain and cystitis both depended on the abnormal position of her uterus, so a ventral suspension was done. Immediately after the operation the patient had a rather profuse haematuria lasting some days. This gradually cleared up, but her pain and frequency remained and her urine continued to show microscopic blood. The bladder, on examination, was found difficult to cleanse, and very intolerant to distention; it would hold no more than $3 \mathrm{oz}$. without giving great pain. The cystoscope showed that the trigone and lateral walls of the bladder were inflamed, thickened and covered with great flakes of muco-pus. With the prism turned upward, a moderate sized stone could be plainly seen. The curious position of the stone was accounted for by the fact that the ventral suspension had pulled up the bladder in such a way as to make it an inverted cone, and that when I had introduced the cystoscope it had slid between the bladder base and the stone, raising the stone from the floor. Litholapaxy was performed by Dr. Kingman the next day. The stone was very soft and had as a nucleus what seemed to be a bit of mucous membrane. The patient went home in a few days entirely relieved of her pain and frequency and with a urine that was clearing up rapidly. Even after this calculus had been seen with the cystoscope, bi-manual examination failed to detect its presence.

CASE VII is one of cancer of the left kidney. The patient was a married woman, fifty-seven years old, seen with Dr. $W^{r}$. A. Brooks, through whose courtesy I report the case.

When the patient was seen in January, 1906, she had a painless haematuria that had persisted for two weeks. Four weeks before she had passed bloody urine at one micturition. There was a vague history of a little pain and of a tender spot in the left loin during the summer of 1905 . The left kidney was not tender and was not definitely enlarged. There were no other localizing symptoms. The urine- was so bloody that it was almost impossible to distinguish any formed elements other than blood globules. The bladder was very tolerant and easily washed clean.

Cystoscopic examination showed an extremely pale but otherwise normal bladder, with rather infrequent jets of what appeared to be pure blood coming from the left ureter. The first time she was examined, the bladder medium could not be kept clear a sufficient time to catheterize the right ureter. The urine from it, however, appeared to be clear. Five days later, the bleeding being a little less, by the use of greater distention I was able to keep the medium clear a suffi- cient length of time to catheterize the right ureter. The right urine had a slight trace of albumin, $1.39 \%$ of urea, a few blood globules (accidental, due to trauma of the catheter), a few leucocytes, a few round, spindle and oblong cells; no casts; in other words, the urine showed a kidney of fair capacity.

The absence of any considerable pain, together with the absence of casts or of any evidence of suppuration, led me to the probable diagnosis of malignant disease, which was heightened by the negative findings of radiography. Somewhat later the bleeding increased and the patient began to have occasional attacks of colic, due to the expulsion of clots.

Owing to Dr. Brooks' departure for Europe, the patient was operated upon by Dr. Mixter Feb. 2. The left kidney, which was removed, showed on its anterior surface near the lower pole, a malignant mass as big as a plum, extending from the cortex to the pelvis. It had apparently not burst through the capsule. The right kidney has shown itself to be perfectly competent and the patient is making a good recovery.

CASE VIII shows another instance of painless haematuria due, however, to a different cause. The patient was a Chinese merchant, aged forty-three, referred to me by Dr. E. M. Greene.

His previous history was unimportant. For three months he had been passing bloody urine, the greater part of the time without pain. For a little while he had had some frequency of micturition. He had noted that there was often no bleeding at night. There was no symptom that gave any hint as to the source of this man's bleeding. Palpation was negative. His urine, which was bright red, contained a considerable number of clots, and no formed element other than normal blood cells were found. He had a moderately tolerant bladder, but the bleeding made it difficult to wash it clean.

Cystoscopic examination, made through a slightly bloody medium, showed a wavy mass with hemorrhagic patches on the right side of the bladder near the right ureteric orifice. This mass is undoubtedly a papillomatous growth of some type for which operation has been advised, but not yet carried out.

In all of the above cases the cystoscopic examination added very definitely to the knowledge that had been obtained by the other methods of study. In Case III this knowledge simply corroborated and added some interesting, but perhaps unimportant, data to what was already known. In the other cases, with the exception of Case VI, in which sounding or digital examination of the bladder would doubtless have given the same result, cystoscopy gave valuable knowledge that could have been acquired in no other way with so little danger or discomfort to the patient.

In the cases of painless haematuria of renal origin, cystoscopy is of particular value, allowing one to tell definitely which kidney the bleeding is from before the other localizing symptoms; pain,tenderness and increased size, have appeared. The great value of this early localization in cases of malignant disease is self evident.

Typhus Fever in Mexico. - Dr. Bernardo Jacoby, of New Orleans, has reached Mexico City to make a study of typhus fever. There have been 139 new cases since March 1, with 32 deaths. The sanitary campaign in the poorer quarters of the city is energetically prosecuted. - American Medicine. 